TRANSACTIONS OF THE

AMERICAN MATHEMATICAL SOCIETY

Volume 350, Number 2, February 1998, Pages 559-569

S $0002-9947(98) 01803-0$

\title{
RELATIVITY OF THE SPECTRUM AND DISCRETE GROUPS ON HYPERBOLIC SPACES
}

\author{
N. MANDOUVALOS
}

\begin{abstract}
We give a simple proof of the analytic continuation of the resolvent
\end{abstract} kernel for a convex cocompact Kleinian group.

\section{INTRODUCTION}

Let us consider hyperbolic manifolds $\Gamma \backslash \mathbb{H}^{n+1}$, where $\Gamma$ is a discrete group acting on the $(n+1)$-dimensional hyperbolic space $\mathbb{H}^{n+1}$. Then the spectrum of the structure, that is, the spectrum of the invariant Laplacian which acts on automorphic functions with respect to $\Gamma$, depends on both $\Gamma$ and $\mathbb{H}^{n+1}$. Let us denote it by $\operatorname{Sp}\left(\Gamma ; \mathbb{H}^{n+1}\right)$. Embedding $\Gamma$ into a higher dimensional hyperbolic space $\mathbb{H}^{m+1}$, $m>n$, one has that $\operatorname{Sp}\left(\Gamma ; \mathbb{H}^{n+1}\right)$ is very different from $\operatorname{Sp}\left(\Gamma ; \mathbb{H}^{m+1}\right)$, despite the fact that $\Gamma$ is kept fixed.

We shall use this phenomenon in order to give a simple proof of the analytic continuation of the resolvent kernel for a convex cocompact Kleinian group. This is known to be a difficult problem. The idea goes back to the program concerning the analytic continuation of the Eisenstein series for Kleinian groups, [2], [3], but has never been published before.

For a different method of the analytic continuation of the resolvent kernel see [5]. The main difference which exists between Mazzeo-Melrose's theory and our own, at least as far as the analytic continuation of the Eisenstein series is concerned, lies in the fact that they cannot produce the functional equation for the Eisenstein series as they do not possess the "Maass-Selberg" relations.

Part of the paper formed the content of a lecture I gave at the third International Congress of Geometry held at the Aristotle University of Thessaloniki, Greece, 16 May to 1 June 1992. I would like to express my warm thanks to the organizing committee for kindly inviting me to give this lecture.

\section{Resolvent Kernel, Eisenstein Series AND the SCATtering Matrix}

Let $\mathbb{H}^{n+1}=\mathbb{R}^{n} \times \mathbb{R}_{+}^{\times}$equipped with the Riemannian metric

$$
d s^{2}=y^{-2}\left(\|d x\|^{2}+d y^{2}\right)
$$

at $w=(x, y), x \in \mathbb{R}^{n}, y:=\operatorname{Im}(w)>0$. This is one of the models of an $(n+1)$-dimensional hyperbolic space. The group of isometries of the structure is $S O(n+1,1)$ and we denote it by $\operatorname{Con}(n+1)$.

Received by the editors August 1, 1995 and, in revised form, December 28, 1995.

1991 Mathematics Subject Classification. Primary 11F72.

(C)1998 American Mathematical Society 
The invariant Laplacian is

$$
L=y^{2}\left(\frac{\partial^{2}}{\partial x_{1}^{2}}+\cdots+\frac{\partial^{2}}{\partial x_{n}^{2}}+\frac{\partial^{2}}{\partial y^{2}}\right)-(n-1) y \frac{\partial}{\partial y}
$$

so that $-L \geq 0$. This is an essentially self-adjoint operator. The action of $\operatorname{Con}(n+1)$ extends by continuity to $\widetilde{\mathbb{R}}^{n}:=\mathbb{R}^{n} \cup\{\infty\}$.

Let $\sigma\left(w_{1}, w_{2}\right):=\left(\left\|x_{1}-x_{2}\right\|^{2}+\left(y_{1}+y_{2}\right)^{2}\right) / 4 y_{1} y_{2}$ be the standard point-pair invariant. It satisfies

$$
\sigma\left(g w_{1}, g w_{2}\right)=\sigma\left(w_{1}, w_{2}\right) \quad \text { for all } g \in \operatorname{Con}(n+1)
$$

and it is related to the hyperbolic distance $\rho\left(w_{1}, w_{2}\right)$ with the formula:

$$
\sigma\left(w_{1}, w_{2}\right)=\frac{1}{2}\left(1+\cosh \rho\left(w_{1}, w_{2}\right)\right) .
$$

Let also

$$
P(w, \zeta)=y /\left(\|x-\zeta\|^{2}+y^{2}\right), \quad w=(x, y) \in \mathbb{H}^{n+1},
$$

$\zeta \in \mathbb{R}^{n}$, be the Poisson kernel. If $j(g, w)$ is the factor of automorphy which is defined by $\operatorname{Im}(g w)=j(g, w) \operatorname{Im}(w), g \in \operatorname{Con}(n+1)$, then one has

$$
P(g w, g \zeta) j(g, \zeta)=P(w, \zeta)
$$

where $j$ has been extended to $\mathbb{R}^{n}$.

The first result that we shall need concerns the resolvent operator and the resolvent kernel.

Let

$$
R(s)=(-L-s(n-s) I)^{-1}
$$

be the resolvent operator. From the spectral theorem for self-adjoint operators $R(s)$ is well defined as a bounded operator on the space $L^{2}\left(\mathbb{H}^{n+1}\right)$, initially for $\operatorname{Re}(s)>n$. See [5, p. 289], and [3]. This is an integral operator and in order to find its kernel we apply the Laplacian operator $-L$ on functions of the point-pair invariant $\sigma\left(w, w^{\prime}\right)$ as functions of the first variable $w$. Hence if $f(w)=\Phi\left(\sigma\left(w, w^{\prime}\right)\right)$ satisfies $-L f=s(n-s) f$, the $\Phi$ satisfies the following hypergeometric equation:

$$
\left(-\sigma^{2}+\sigma\right) \Phi^{\prime \prime}(\sigma)-(n+1)\left(\sigma-\frac{1}{2}\right) \Phi^{\prime}(\sigma)-s(n-s) \Phi(\sigma)=0 .
$$

One solution of this equation is

$$
\begin{aligned}
& r\left(w, w^{\prime} ; s\right) \\
& \quad=\frac{\pi^{-n / 2}}{2} 4^{-s} \frac{\Gamma(s)}{\left(s-\frac{n}{2}\right) \Gamma\left(s-\frac{n}{2}\right)} \sigma\left(w, w^{\prime}\right)^{-s}{ }_{2} F_{1}\left(s, s+\frac{1}{2}-\frac{n}{2} ; 2 s-n+1 ; \sigma^{-1}\right)
\end{aligned}
$$

where ${ }_{2} F_{1}(\cdot, \cdot ; \cdot ; \cdot)$ is the Gauss hypergeometric function. This is the resolvent kernel [3, p. 213], [7, pp. 419-420], [8, p. 69] and it has an analytic continuation for $\operatorname{Re}(s) \geq \frac{n}{2}$. It behaves as $\sigma\left(w, w^{\prime}\right) \rightarrow \infty$ as

$$
\frac{\pi^{-n / 2}}{2} 4^{-s} \frac{\Gamma(s)}{\left(s-\frac{n}{2}\right) \Gamma\left(s-\frac{n}{2}\right)} \sigma\left(w, w^{\prime}\right)^{-s} .
$$

On the other hand we have the relation

$$
\begin{gathered}
\int_{\mathbb{R}^{n}} P(w, \zeta)^{s} P\left(w^{\prime}, \zeta\right)^{n-s} d m(\zeta)=\pi^{n / 2} \frac{\Gamma\left(\frac{n}{2}-s\right)}{\Gamma(n-s)} 4^{-s} \sigma\left(w, w^{\prime}\right)^{-s} \\
\times{ }_{2} F_{1}\left(s, s+\frac{1}{2}-\frac{n}{2} ; 2 s-n+1 ; \sigma^{-1}\right)+(s \rightarrow n-s),
\end{gathered}
$$


see [4, p. 82], where $(s \rightarrow n-s)$ is equal to the first term of the right-hand side with $s$ replaced by $n-s$. From this relation and the relation which gives the resolvent kernel we get

$$
\begin{array}{rl}
\int_{\mathbb{R}^{n}} & P(w, \zeta)^{s} P\left(w^{\prime}, \zeta\right)^{n-s} d m(\zeta) \\
\quad=2 \pi^{n}\left(s-\frac{n}{2}\right) \frac{\Gamma\left(s-\frac{n}{2}\right) \Gamma\left(\frac{n}{2}-s\right)}{\Gamma(s) \Gamma(n-s)}\left(r\left(w, w^{\prime} ; s\right)-r\left(w, w^{\prime} ; n-s\right)\right), \quad \operatorname{Re}(s) \geq n .
\end{array}
$$

Now let $\Gamma$ be a discrete subgroup of $\operatorname{Con}(n+1)$. We make the assumption that $\Gamma$ is geometrically finite with a fundamental region of infinite hyperbolic measure and is convex cocompact. We further assume that $\Gamma$ contains no elements of finite order. These assumptions guarantee that $M=\Gamma \backslash \mathbb{H}^{n+1}$ is a Riemannian manifold. Moreover it carries the above-defined Laplace operator $L$.

We denote by $\Omega(\Gamma) \subseteq \widetilde{\mathbb{R}}^{n}$ the region of discontinuity of $\Gamma$ and by $L(\Gamma)=\widetilde{\mathbb{R}}^{n}-$ $\Omega(\Gamma)$ the limit set of $\Gamma$.

Let

$$
\delta(\Gamma):=\inf \left\{s>0: \sum_{\gamma \in \Gamma} \sigma\left(\gamma w_{1}, w_{2}\right)^{-s}<+\infty\right\}
$$

be the exponent of convergence of the Poincaré series. This is an invariant. It does not depend on the choice of $w_{1}, w_{2}$. It is known that $0 \leq \delta(\Gamma) \leq n$. Moreover $\delta(\Gamma)$ is equal to the Hausdorff dimension of $L(\Gamma)$. This is a theorem due to S. J. Patterson and D. Sullivan; [6], [10].

The resolvent kernel for the group $\Gamma$, that is, the kernel of the operator $(-L-s(n-s) I)^{-1}$ acting on functions on $\Gamma \backslash \mathbb{H}^{n+1}$, is given by the expression:

$$
R\left(w, w^{\prime} ; s\right)=\sum_{\gamma \in \Gamma} r\left(w, \gamma w^{\prime} ; s\right), \quad \operatorname{Re}(s)>\delta(\Gamma),
$$

where $r\left(w, w^{\prime} ; s\right)$ is the resolvent kernel for the trivial group acting on $\mathbb{H}^{n+1}$, as it was described above. See [3, Proposition 4.3].

If $w \in \mathbb{H}^{n+1}, \zeta \in \Omega(\Gamma)$ and $s \in \mathbb{C}$, then for $\operatorname{Re}(s)>\delta(\Gamma)$ we define the Eisenstein series

$$
E(w, \zeta ; s)=\sum_{\gamma \in \Gamma} P(\gamma w, \zeta)^{s}
$$

This is an eigenfunction of the Laplacian

$$
-L E(w, \zeta ; s)=s(n-s) E(w, \zeta ; s) .
$$

We also define the " $S$-matrix"

$$
S(\zeta, \eta ; s)=\sum_{\gamma \in \Gamma} \frac{j(\gamma, \zeta)^{s}}{\|\gamma(\zeta)-\eta\|^{2 s}}
$$

where $\zeta, \eta \in \Omega(\Gamma)$ and $\operatorname{Re}(s)>\delta(\Gamma)$.

The "S-matrix" describes the normalized boundary behaviour of the Eisenstein series:

$$
S(\eta, \zeta ; s)=\lim _{w \rightarrow \eta} y^{-s} E(w, \zeta ; s)
$$


The terminology "S-matrix" is justified by the fact that $S(\cdot, \cdot ; s)$ participates in the formulation of the functional equation for the Eisenstein series, which has a quantum mechanical interpretation coming from scattering theory [2].

Now if $\Gamma$ is such that $\delta(\Gamma)<n / 2$, then the group $\Gamma$ has a purely continuous spectrum which can be described by the above Eisenstein series [3]. These series have an analytic continuation in the whole complex plane and the following functional equation holds:

$$
\begin{array}{r}
\int_{\Gamma \backslash \Omega(\Gamma)} E(w, \zeta ; s) S(\zeta, \eta ; n-s) d m(\zeta) \\
=\pi^{n / 2} \frac{\Gamma\left(s-\frac{n}{2}\right)}{\Gamma(s)} E(w, \eta ; n-s),
\end{array}
$$

$[2],[4]$.

\section{The Embedding into higher Dimensional hyperbolic StruCtures}

Now for $0<\theta<\pi$ we introduce the map

$$
\begin{gathered}
J_{\theta}(n+1)=: \mathbb{H}^{n+1} \rightarrow \mathbb{H}^{n+2}, \\
\mathbb{H}^{n+1} \ni(x, y) \rightarrow((x, y \cos \theta), y \sin \theta) \in \mathbb{H}^{n+2} .
\end{gathered}
$$

We may extend this map for $\theta=0$ and $\theta=\pi$ as well. Indeed one observes that

$$
J_{0}^{(n+1)}(x, y)=((x, y), 0) .
$$

Moreover the set $\left\{((x, y), 0): y>0, x \in \mathbb{R}^{n}\right\}$ can be also identified with $\mathbb{H}^{n+1}$. Thus

$$
J_{0}^{(n+1)}\left(\mathbb{H}^{n+1}\right)=\widetilde{\mathbb{R}}_{+}^{n+1} \subset \partial \mathbb{H}^{n+2} .
$$

Similarly

$$
J_{\pi}\left(\mathbb{H}^{n+1}\right)=\widetilde{\mathbb{R}}_{-}^{n+1} \subset \partial \mathbb{H}^{n+2},
$$

and this can be identified with $\mathbb{H}_{-}^{n+1}$, the lower half space.

Consequently the above map is extended for $0 \leq \theta \leq \pi$.

One can actually make several such similar choices of the map $j_{\theta}^{(n+1)}$ which depend on the parameter $\theta$ in slightly different ways.

The map $j_{\theta}^{(n+1)}$ has the property that there is an embedding

$$
\widetilde{J}_{n+1}: \operatorname{Con}(n+1) \rightarrow \operatorname{Con}(n+2),
$$

such that

$$
j_{\theta}^{(n+1)}(g w)=\widetilde{J}_{n+1}(g) \cdot j_{\theta}^{(n+1)}(w), \quad g \in \operatorname{Con}(n+1) .
$$

Next let us perform $m$ such embeddings

$$
\mathbb{H}^{n+1} \stackrel{j_{\theta_{1}}^{(n+1)}}{\longrightarrow} \mathbb{H}^{n+2} \rightarrow \cdots \stackrel{j_{\theta_{m}}^{(n+m)}}{\longrightarrow} \mathbb{H}^{n+1+m} .
$$

We denote by

$$
\theta(m):=\left(\theta_{1}, \theta_{2}, \ldots, \theta_{m}\right), \quad I_{\theta(m)}^{(m)}:=j_{\theta_{m}}^{(n+m)} \cdots j_{\theta_{1}}^{(n+1)},
$$

and $\widetilde{J}:=\widetilde{J}_{n+m} \cdots \widetilde{J}_{n+1}$, where

$$
\operatorname{Con}(n+1) \stackrel{\widetilde{J}_{n+1}}{\longrightarrow} \operatorname{Con}(n+2) \rightarrow \cdots \stackrel{\widetilde{J}_{n+m}}{\longrightarrow} \operatorname{Con}(n+1+m) .
$$


Let $\Gamma$ be a discrete subgroup of $\operatorname{Con}(n+1)$ which is convex cocompact. It is known that $m_{n}(L(\Gamma))=0$ [11], where $m_{n}$ is the $n$-dimensional Lebesgue measure. Moreover $\Gamma \backslash \Omega(\Gamma)$ is a compact manifold. The following three facts are of vital importance.

First we observe that $\widetilde{J}_{n+1}(\Gamma)$ is a discrete subgroup of $\operatorname{Con}(n+2)$. Moreover by repeating this map we can embed $\Gamma$ into $\operatorname{Con}(m+1)$ for any $m>n$.

Second the set $\left\{J_{\theta}^{(n+1)}(w): 0<\theta<\pi, w \in F\right\}$ is a fundamental domain for $J_{n+1}(\Gamma)$ if $F$ is one for $\Gamma$.

Third $L(\widetilde{J}(\Gamma))=J_{0}^{(n+1)}(L(\Gamma)) \equiv L(\Gamma)$.

This last property is very important and it simply says that the limit set of $\Gamma$ does not change under such an embedding of the original hyperbolic structure into a higher one. This in particular implies that $\delta(\Gamma)$ does not change under such an embedding, since it is equal to the Hausdorff dimension of $L(\Gamma)$.

Let us now produce the expressions of the Eisenstein series and " $S$-matrix" under such a multiple embedding of the original group.

Lemma 1 (The case of one only embedding). Let

$$
J_{\theta}^{(n+1)}: \mathbb{H}^{n+1} \rightarrow \mathbb{H}^{n+2}
$$

be the embedding we introduced above.

Then the Poisson kernel in $\mathbb{H}^{n+2}$ in terms of elements of the space $\mathbb{H}^{n+1}$ is given by the formula

$$
\begin{aligned}
& P\left(j_{\theta}^{(n+1)}(w), j_{0}^{(n+1)}\left(w^{\prime}\right)\right) \\
& \quad=\frac{1}{4} \operatorname{Im}\left(w^{\prime}\right)^{-1} \sin \theta \cdot\left[\sigma\left(w, w^{\prime}\right)-\frac{1}{2}(1+\cos \theta)\right]^{-1}
\end{aligned}
$$

where $w, w^{\prime} \in \mathbb{H}^{n+1}$ and $\sigma\left(w, w^{\prime}\right)$ is the standard point-pair invariant in $\mathbb{H}^{n+1}$.

The proof is trivial.

Lemma 2 (The case of $m$-embeddings). Let

$$
\mathbb{H}^{n+1} \stackrel{j_{\theta_{1}}^{(n+1)}}{\longrightarrow} \mathbb{H}^{n+2} \rightarrow \cdots \stackrel{j_{\theta_{m}}^{(n+m)}}{\longrightarrow} \mathbb{H}^{n+1+m}
$$

be a sequence of $m$-embeddings. Let $w, w^{\prime} \in \mathbb{H}^{n+1}$ and

$$
\beta_{m}:=1-\frac{1}{2}\left(1-\cos \theta_{m}\right) \sin ^{2} \theta_{1} \cdots \sin ^{2} \theta_{m-1},
$$

where $\theta_{0}=\frac{\pi}{2}$.

Let also

$$
\begin{aligned}
A_{m}(w) & :=j_{\theta_{m}}^{(n+m)} I_{\theta(m-1)}^{(m-1)}(w) \quad \text { and } \\
B_{m}\left(w^{\prime}\right) & :=j^{(n+m)} I_{\theta(m-1)}^{(m-1)}\left(w^{\prime}\right) .
\end{aligned}
$$

Then the following hold:

(i) The Poisson kernel in $\mathbb{H}^{n+1+m}$ is given in terms of elements of the space $\mathbb{H}^{n+1}$ by the formula

$$
P\left(A_{m}(w), B_{m}\left(w^{\prime}\right)\right)=4^{-1} \operatorname{Im}\left(w^{\prime}\right)^{-1} \sin \theta_{1} \cdots \sin \theta_{m}\left[\sigma\left(w, w^{\prime}\right)-\beta_{m}\right]^{-1} .
$$


(ii) The Eisenstein series in $\mathbb{H}^{n+1+m}$ in terms of elements of the space $\mathbb{H}^{n+1}$ are given by

$$
\begin{aligned}
& E\left(A_{m}(w), B_{m}\left(w^{\prime}\right) ; s\right) \\
& \quad=4^{-s} \operatorname{Im}\left(w^{\prime}\right)\left(\sin \theta_{1} \cdots \sin \theta_{m}\right)^{s} \sum_{\gamma \in \Gamma}\left[\sigma\left(\gamma w, w^{\prime}\right)-\beta_{m}\right]^{-s} .
\end{aligned}
$$

(iii) The "S-matrix" which is attached to the space $\Omega\left(\Gamma_{\mathbb{H} n+1+m}\right)$ is given by

$$
\begin{aligned}
& S\left(B_{m}\left(w^{\prime}\right), B_{m}\left(w^{\prime \prime}\right) ; s\right) \\
& \quad=4^{-s} \operatorname{Im}\left(w^{\prime}\right)^{-s} \operatorname{Im}\left(w^{\prime \prime}\right)^{-s} \sum_{\gamma \in \Gamma}\left[\sigma\left(\gamma w^{\prime}, w^{\prime \prime}\right)-1\right]^{-s},
\end{aligned}
$$

where $w^{\prime \prime} \in \mathbb{H}^{n+1}$.

Proof. (i) First we are going to show the following identity:

$$
\left\|A_{m}(w)-B_{m}\left(w^{\prime}\right)\right\|^{2}=4 y y^{\prime}\left[\sigma\left(w, w^{\prime}\right)-\beta_{m}\right],
$$

where $\|\cdot\|$ denotes the Euclidean norm.

Let

$$
w=(x, y), \quad w^{\prime}=\left(x^{\prime}, y^{\prime}\right) \in \mathbb{H}^{n+1} .
$$

By a careful consideration of the embeddings it is not difficult to verify the following identities:

$$
\begin{aligned}
& A_{1}(w)=\left(\left(x, y \cos \theta_{1}\right), y \sin \theta_{1}\right), \quad B_{1}\left(w^{\prime}\right)=\left(\left(x^{\prime}, y^{\prime}\right), 0\right) \equiv\left(x^{\prime}, y^{\prime}\right), \\
& A_{m-1}(x)=\left(\left(X, y \sin \theta_{1} \sin \theta_{2} \cdots \sin \theta_{m-2} \cos \theta_{m-1}\right), y \sin \theta_{1} \cdots \sin \theta_{m-1}\right), \\
& B_{m-1}\left(w^{\prime}\right)=\left(X^{\prime}, y^{\prime} \sin \theta_{1} \cdots \sin \theta_{m-2}\right),
\end{aligned}
$$

where

$$
X=X\left(x, y ; \theta_{1}, \ldots, \theta_{m-2}\right)
$$

is an explicit function of the above arguments, which is independent of $\theta_{m-1}$ and $X^{\prime}$ is given by $X$ with $x, y$ replaced by $x^{\prime}, y^{\prime}$ respectively. (We do not however need the exact formulae of $X$ and $X^{\prime}$.)

Similarly we have:

$$
\begin{aligned}
A_{m}(w)=\left(\left(\left(X, y \sin \theta_{1} \cdots \sin \theta_{m-2} \cos \theta_{m-1}\right),\right.\right. \\
\left.\left.y \sin \theta_{1} \cdots \sin \theta_{m-1} \cos \theta_{m}\right), y \sin \theta_{1} \cdots \sin \theta_{m}\right),
\end{aligned}
$$

and

$$
B_{m}\left(w^{\prime}\right)=\left(\left(X^{\prime}, y^{\prime} \sin \theta_{1} \cdots \sin \theta_{m-2} \cos \theta_{m-1}\right), y^{\prime} \sin \theta_{1} \cdots \sin \theta_{m-1}\right) .
$$

Having obtained the above expressions it is an easy matter to verify the identity

$$
\begin{aligned}
& \left\|A_{m}(w)-B_{m}\left(w^{\prime}\right)\right\|^{2}=\left\|A_{m-1}(w)-B_{m-1}\left(w^{\prime}\right)\right\|^{2}+\sin ^{2} \theta_{1} \cdots \sin ^{2} \theta_{m-2} \\
& \quad \cdot \cos ^{2} \theta_{m-1}\left(y-y^{\prime}\right)^{2}+\sin ^{2} \theta_{1} \cdots \sin ^{2} \theta_{m-1}\left(y \cos \theta_{m}-y^{\prime}\right)^{2} \\
& \quad+y^{2} \sin ^{2} \theta_{1} \cdots \sin ^{2} \theta_{m}-\sin ^{2} \theta_{1} \cdots \sin ^{2} \theta_{m-2}\left(y \cos \theta_{m-1}-y^{\prime}\right)^{2} \\
& \quad-y^{2} \sin ^{2} \theta_{1} \cdots \sin ^{2} \theta_{m-1} .
\end{aligned}
$$

For the rest of the proof we apply an induction argument. We know by Lemma 1 that

$$
\left\|A_{1}(w)-B_{1}\left(w^{\prime}\right)\right\|^{2}=4 y y^{\prime}\left[\sigma\left(w, w^{\prime}\right)-1+\frac{1}{2}\left(1-\cos \theta_{1}\right)\right] .
$$


Let us now assume that $(*)$ holds for $m-1$, that is,

$$
\begin{aligned}
& \left\|A_{m-1}(w)-B_{m-1}\left(w^{\prime}\right)\right\|^{2} \\
& \quad=4 y y^{\prime}\left[\sigma\left(w, w^{\prime}\right)-1+\frac{1}{2}\left(1-\cos \theta_{m-1}\right) \sin ^{2} \theta_{1} \cdots \sin ^{2} \theta_{m-2}\right] .
\end{aligned}
$$

Then substituting this expression of $\left\|A_{m-1}(w)-B_{m-1}\left(w^{\prime}\right)\right\|^{2}$ into $(* *)$ we obtain the result after a trivial calculation.

Having obtained $(*)$ we observe that

$$
P\left(A_{m}(w), B_{m}\left(w^{\prime}\right)\right)=\operatorname{Im}(w) \cdot \sin \theta_{1} \cdots \sin \theta_{m} /\left\|A_{m}(w)-B_{m}\left(w^{\prime}\right)\right\|^{2} .
$$

Now substituting in this formula the expression of $\left\|A_{m}(w)-B_{m}\left(w^{\prime}\right)\right\|^{2}$ from (*) we obtain (i).

(ii) The expression which gives the Eisenstein series follows now easily.

Indeed one observes that

$$
A_{m}(\gamma w)=\widetilde{J}(\gamma) A_{m}(w)
$$

where

$$
\widetilde{J}(\gamma):=\widetilde{J}_{m+n} \cdots \widetilde{J}_{n+1}(\gamma)
$$

Hence

$$
\begin{aligned}
& E\left(A_{m}(w), B_{m}\left(w^{\prime}\right) ; s\right)=\sum_{\gamma \in \Gamma} P\left(\widetilde{J}(\gamma) A_{m}(w), B_{m}\left(w^{\prime}\right)\right)^{s} \\
& \quad=\sum_{\gamma \in \Gamma} P\left(A_{m}(\gamma w), B_{m}\left(w^{\prime}\right)\right)^{s} \\
& \quad=4^{-s} \operatorname{Im}\left(w^{\prime}\right)^{-s}\left[\sin \theta_{1} \cdots \sin \theta_{m}\right]^{s} \cdot \sum_{\gamma \in \Gamma}\left[\sigma\left(\gamma w, w^{\prime}\right)-\beta_{m}\right]^{-s} .
\end{aligned}
$$

(iii) The expression of the " $S$-matrix" follows now in an obvious way.

This completes the proof of Lemma 2.

\section{The AnALYTIC CONTINUATION}

In this section we are going to prove the analytic continuation of the resolvent kernel by using the embeddings into higher dimensional hyperbolic structures and the "relativity" of the spectrum.

In fact the following theorem holds.

Theorem. Let $\Gamma$ be a convex cocompact Kleinian group acting on $\mathbb{H}^{n+1}$. Then the resolvent kernel has an analytic continuation in the whole complex plane as a meromorphic function.

For the proof we need the following lemma.

Lemma. Let $\Gamma$ be a convex cocompact Kleinian group acting on $\mathbb{H}^{m+1}$, where $\delta(\Gamma)<m / 2$. Then the Eisenstein series $E(w, \zeta ; s), w \in \mathbb{H}^{m+1}, \zeta \in \Omega(\Gamma) \subseteq \widetilde{\mathbb{R}}^{m}$, have an analytic continuation in the whole complex plane as a meromorphic function of $s$ and the corresponding functional equation holds:

$$
E\left(w, S_{m-s}(f), s\right)=\pi^{m / 2} \frac{\Gamma(s-m / 2)}{\Gamma(s)} E(w, f, m-s) .
$$


Proof. Since $\delta(\Gamma)<m / 2$, the group $\Gamma$ acting on $\mathbb{H}^{m+1}$ has a purely continuous spectrum. Then this lemma is part (ii) of Theorem 5.1 on p. 233 of [3]. The exact form of the functional equation on $\mathbb{H}^{m+1}$ is in $[4$, p. 84].

Let us now return to the proof of the above theorem.

First, we choose a natural number $m, m>n$, such that $\delta(\Gamma)<m / 2$.

Second, we make $\Gamma$ act on the hyperbolic space $\mathbb{H}^{m+1}$ by using the stepwise embedding of $\mathbb{H}^{n+1}$ into $\mathbb{H}^{m+1}$ in the way we described in $\S 3$. Then $\Gamma$ acting on $\mathbb{H}^{m+1}$ has a purely continuous spectrum and the method which has been developed in [3], or more simply the above lemma applies. Consequently the corresponding Eisenstein series in $\mathbb{H}^{m+1}$ have an analytic continuation in the whole complex plane as a meromorphic function of $s$. This implies from the corresponding expression of the Eisenstein series in Lemma 2, (ii), that the series of the form

$$
K\left(w, w^{\prime} ; s\right):=\sum_{y \in \Gamma}\left[\sigma\left(y w, w^{\prime}\right)-\beta_{m}\right]^{-s},
$$

where $w, w^{\prime} \in \mathbb{H}^{n+1}$, have an analytic continuation on the whole complex plane. Thus

$$
\begin{aligned}
K^{*}\left(w, w^{\prime} ; s\right) & =\frac{1}{2} \pi^{-n / 2} 4^{-s} \frac{\Gamma(s)}{\left(s-\frac{n}{2}\right) \Gamma\left(s-\frac{n}{2}\right)} K\left(w, w^{\prime} ; s\right) \\
& =\frac{1}{2} \pi^{-n / 2} 4^{-s} \frac{\Gamma(s)}{\left(s-\frac{n}{2}\right) \Gamma\left(s-\frac{n}{2}\right)} \sum_{\gamma \in \Gamma} \sigma\left(\gamma w, w^{\prime}\right)^{-s}+N\left(w, w^{\prime} ; s\right),
\end{aligned}
$$

where $N\left(w, w^{\prime} ; s\right)$ converges for $\operatorname{Re}(s)>\delta(\Gamma)-1$.

Moreover from the expression of the resolvent kernel $R\left(w, w^{\prime} ; s\right)$ for $\Gamma$ acting on $\mathbb{H}^{n+1}$, Lemma 2, we have

$$
R\left(w, w^{\prime} ; s\right)=\pi^{-n / 2} \frac{4^{-s} \Gamma(s)}{\left(s-\frac{n}{2}\right) \Gamma\left(s-\frac{n}{2}\right)} \sum_{\gamma \in \Gamma} \sigma\left(\gamma w, w^{\prime}\right)^{-s}+\Lambda\left(w, w^{\prime} ; s\right),
$$

where $\Lambda\left(w, w^{\prime} ; s\right)$ converges for $\operatorname{Re}(s)>\delta(\Gamma)-1$, since

$$
{ }_{2} F_{1}\left(\sigma\left(\gamma w, w^{\prime}\right)^{-1}\right)=1+O\left(\sigma\left(\gamma w, w^{\prime}\right)^{-1}\right) \quad \text { as } \sigma \rightarrow \infty,
$$

where ${ }_{2} F_{1}\left(\sigma^{-1}\right):={ }_{2} F_{1}\left(s, s+\frac{1}{2}-\frac{n}{2} ; 2 s-n+1 ; \sigma^{-1}\right)$.

Consequently the difference

$$
R\left(w, w^{\prime} ; s\right)-K^{*}\left(w, w^{\prime} ; s\right)=\Lambda\left(w, w^{\prime} ; s\right)-N\left(w, w^{\prime} ; s\right)
$$

converges for $\operatorname{Re}(s)>\delta(\Gamma)-1$, since both terms on the right-hand side do.

But as we have proved, $K\left(w, w^{\prime} ; s\right)$ has an analytic continuation in the whole complex plane as a meromorphic function of $s$; this obviously holds for $K^{*}\left(w, w^{\prime} ; s\right)$ as well.

This then implies that the resolvent kernel $R\left(w, w^{\prime} ; s\right)$ has an analytic continuation in the region $\operatorname{Re}(s)>\delta(\Gamma)-1$.

Repeating the above argument we obtain the analytic continuation for $R\left(w, w^{\prime} ; s\right)$ in the whole complex plane, as follows.

Since $R\left(w, w^{\prime} ; s\right)$ behaves like $\sum_{\gamma \in \Gamma} \sigma\left(\gamma w, w^{\prime}\right)^{-s}$ as $\sigma \rightarrow \infty$, the fact that $R\left(w, w^{\prime} ; s\right)$ has an analytic continuation for $\operatorname{Re}(s)>\delta(\Gamma)-1$ implies that the series $\sum_{\gamma \in \Gamma} \sigma\left(\gamma w, w^{\prime}\right)^{-s}$ have an analytic continuation for $\operatorname{Re}(s)>\delta(\Gamma)-1$. Therefore the series $\sum_{\gamma \in \Gamma} \sigma\left(\gamma w, w^{\prime}\right)^{-s-1}$ and subsequently both $N\left(w, w^{\prime} ; s\right)$ and $\Lambda\left(w, w^{\prime} ; s\right)$ have an analytic continuation in the region $\operatorname{Re}(s)>\delta(\Gamma)-2$, which in turn implies the 
analytic continuation of $R\left(w, w^{\prime} ; s\right)$ for $\operatorname{Re}(s)>\delta(\Gamma)-2$. Repeating the above argument we obtain after a finite number of steps the analytic continuation of $R\left(w, w^{\prime} ; s\right)$ for $s$ in any compact subset of $\mathbb{C}$. This completes the proof of the theorem.

Corollary. The Eisenstein series have an analytic continuation in the whole complex plane and they satisfy the functional equation of the lemma.

The functional equation follows from the validity of the second "Maass-Selberg" relation [4].

\section{A VARiety of "FunCtional equations"}

In this section we are going to examine the following rather peculiar phenomenon.

Let $\Gamma$ be a convex cocompact Kleinian group acting on $\mathbb{H}^{n+1}$. Then we can make $\Gamma$ act on all higher dimensional hyperbolic spaces $\mathbb{H}^{n+m+1}$ in the way we described in $\S 3$. At each pair $\left(\Gamma, \mathbb{H}^{n+1}\right)$, that is, $\Gamma$ as a discrete group on $\mathbb{H}^{n+1}$, corresponds a functional equation which is satisfied by the corresponding Eisenstein series. Bear in mind that the group $\Gamma$ remains the same.

Now the question arises naturally. Is there a variety of functional equations or what is really going on?

It turns out that only the functional equation in the original space $\mathbb{H}^{n+1}$ is a genuine functional equation for Eisenstein series, while all other equations in the spaces $\mathbb{H}^{n+m+1}, m \geq 1$, have a different structure.

To be more precise let us recall the functional equation in $\mathbb{H}^{n+1}$ :

$$
\int_{\Gamma \backslash \Omega\left(\Gamma_{\mathbb{H}^{n+1}}\right)} E(w, \zeta ; s) S(\zeta, \eta ; n-s) d m(\zeta)=\pi^{n / 2} \frac{\Gamma\left(s-\frac{n}{2}\right)}{\Gamma(s)} E(w, \eta ; n-s),
$$

(see [4, p. 84]), which is a genuine functional equation for Eisenstein series.

Let us now make $\Gamma$ act on $\mathbb{H}^{n+m+1}$ after $m$-embeddings. Then using the formulae which give the Eisenstein series on $\mathbb{H}^{n+m+1}$ in terms of elements of the space $\mathbb{H}^{n+1}$, we obtain the corresponding functional equation for the space $\mathbb{H}^{n+m+1}$ :

$$
\begin{aligned}
& \int_{\Gamma \backslash \Omega\left(\Gamma_{\mathbb{H}^{n} n+1+m}\right)} E\left(A_{m}(w), B_{m}\left(w^{\prime}\right) ; s\right) S\left(B_{m}\left(w^{\prime}\right), B_{m}\left(w^{\prime \prime}\right) ; n+m-s\right) d m_{n+m}\left(w^{\prime}\right) \\
& =\pi^{(n+m) / 2} \frac{\Gamma\left(s-\frac{n+m}{2}\right)}{\Gamma(s)} E\left(A_{m}(w), B_{m}\left(w^{\prime \prime}\right) ; n+m-s\right),
\end{aligned}
$$

where $w, w^{\prime}, w^{\prime \prime} \in \mathbb{H}^{n+1}, \Omega\left(\Gamma_{\mathbb{H}^{n+1+m}}\right) \subseteq \widetilde{\mathbb{R}}^{n+m}$.

Now substituting into this functional equation the Eisenstein series and " $S$ matrix" with the expressions we have found in $\S 3$, in terms of elements of the space $\mathbb{H}^{n+1}$ we get after a trivial calculation:

$$
\begin{gathered}
\int_{\Gamma \backslash \Omega\left(\Gamma_{\mathbb{H} n+1+m}\right)} \sum_{\gamma \in \Gamma}\left[\sigma\left(\gamma w, w^{\prime}\right)-\beta_{m}\right]^{-s} \cdot \sum_{\gamma \in \Gamma}\left[\sigma\left(\gamma w^{\prime}, w^{\prime}\right)-1\right]^{-(n+m-s)} \\
\cdot \operatorname{Im}\left(w^{\prime}\right)^{-(n+m)} d m_{n+m}\left(w^{\prime}\right) \\
=\pi^{(n+m) / 2} \frac{\Gamma\left(s-\frac{n+m}{2}\right)}{\Gamma(s)} 4^{s} \cdot\left[\sin \theta_{1} \cdots \sin \theta_{m}\right]^{n+m-2 s} \\
\cdot \sum_{\gamma \in \Gamma}\left[\sigma\left(\gamma w, w^{\prime \prime}\right)-\beta_{m}\right]^{-(n+m-s)} .
\end{gathered}
$$


Therefore the functional equation for the group $\Gamma$ acting on $\mathbb{H}^{n+1+m}$ now becomes a relation between point-pair invariants.

\section{The Selberg transform of the Kernels}

In this section we are going to evaluate the Selberg transform of the kernels $\left[\sigma\left(w, w^{\prime}\right)-\beta\right]^{-s}$ introduced in $\S 5$.

This kernel is a point-pair invariant. Therefore it defines an integral operator which commutes with the invariant hyperbolic Laplacian [1], [9].

Since $y(w)^{t}$ is an eigenfunction of the Laplacian, the Selberg theory implies that there exists a function $h_{s}(t)$ such that

$$
\int_{\mathbb{H}^{n+1}}\left(\sigma\left(w, w^{\prime}\right)-\beta\right)^{-s} y(w)^{t} d \sigma(w)=h_{s}(t) y\left(w^{\prime}\right)^{t} .
$$

The function $h_{s}(t)$, which is a function of the complex variable $t$, is determined uniquely by the kernel of the operator.

We have

$$
h_{s}(t)=\int_{\mathbb{H}^{n+1}}\left(\sigma\left(w, w^{\prime}\right)-\beta\right)^{-s}\left[\frac{y(w)}{y\left(w^{\prime}\right)}\right]^{t} d \sigma(w) .
$$

Let

$$
w=(x, y), \quad w^{\prime}=\left(x^{\prime}, y^{\prime}\right) \in \mathbb{H}^{n+1} .
$$

Then by making the substitutions:

$$
\begin{aligned}
& \text { first: } x-x^{\prime}=\left(y+y^{\prime}\right) h, \\
& \text { second: } l=\frac{y}{y^{\prime}} \text { and } \\
& \text { third: } z=\frac{l+1}{\sqrt{(l+1)^{2}-4 l \beta}} \cdot h,
\end{aligned}
$$

we get

$$
h_{s}(t)=\int_{\mathbb{R}^{n}}\left(|\xi|^{2}+1\right)^{-s} d m(\xi) \cdot \int_{0}^{\infty}\left[(l+1)^{2}-4 l \beta\right]^{\frac{n}{2}-s} l^{s+t-n-1} d l .
$$

The first integral can be evaluated in terms of $\Gamma$-functions. Thus we obtain

$$
h_{s}(t)=\pi^{n / 2} \frac{\Gamma\left(s-\frac{n}{2}\right)}{\Gamma(s)} \int_{0}^{\infty}\left[(l+1)^{2}-4 l \beta\right]^{n / 2-s} l^{s+t-n-1} d l .
$$

By making the substitution $y=l /(l+1)$ the above can be also written as

$$
h_{s}(t)=\pi^{n / 2} \frac{\Gamma\left(s-\frac{n}{2}\right)}{\Gamma(s)} \int_{0}^{1}[1-4 y \beta(1-y)]^{n / 2-s}(1-y)^{s-t-1} y^{s+t-n-1} d y .
$$

Finally, one can use hypergeometric functions and investigate the behaviour of $h_{s}(t)$ as $\beta \rightarrow 1$. Indeed one gets

$$
\begin{aligned}
h_{s}(t) & \underset{\beta \rightarrow 1}{\longrightarrow} \pi^{n / 2} \frac{\Gamma\left(s-\frac{n}{2}\right)}{\Gamma(s)} \int_{0}^{1} y^{s+t-n-1}(1-y)^{s-t-1}(1-2 y)^{n-2 s} d y \\
& =\pi^{n / 2} \frac{\Gamma\left(s-\frac{n}{2}\right)}{\Gamma(s)} \frac{\Gamma(s+t-n) \Gamma(s-t)}{\Gamma(2 s-n)}{ }_{2} F_{1}(2 s-n, s+t-n ; 2 s-n ; 2),
\end{aligned}
$$

provided that $\operatorname{Re}(s)>\operatorname{Re}(t)$ and $\operatorname{Re}(s+t)>n$.

The function ${ }_{2} F_{1}(\cdot, \cdot ; \cdot ; \cdot)$ is the Gauss hypergeometric function. 


\section{REFERENCES}

[1] T. Kubota, Elementary theory of Eisenstein series, Kodansha, Tokyo, New York, 1973. MR 55:2759

[2] N. Mandouvalos, The theory of Eisenstein series for Kleinian groups, Contemporary Mathematics, V. 53, (1986), 357-370. MR 88f: 11046

[3] __ Spectral theory and Eisenstein series for Kleinian groups, Proc. London Math. Soc. (3) 57 (1988), 209-238. MR 89h:58201

[4] _ Scattering operator, Eisenstein series, inner product formula and "Maass-Selberg" relations for Kleinian groups, Memoirs Amer. Math. Society, V. 78, No. 400, (1989), 1-87. MR 90c: 11036

[5] R. R. Mazzeo and R. B. Melrose, Meromorphic extension of the resolvent on complete spaces with asymptotically constant negative curvature, J. Funct. Anal. 75 (1987), 260-310. MR 89c:58133

[6] S. J. Patterson, The limit set of a Fuchsian group, Acta Math. 136 (1976), 241-273. MR 56: 8841

[7] — The Selberg zeta function of a Kleinian group, Number Theory, Trace Formulas and Discrete Groups, Symposium in Honor of Atle Selberg, Oslo, Norway, July 14-21, Academic Press, 1987, 409-441. MR 91c:11029

[8] P. A. Perry, The Laplace operator on a hyperbolic manifold, II. Eisenstein series and the scattering matrix, J. Reine Angew. Math. 398 (1989), 67-91. MR 90g:58138

[9] A. Selberg, Harmonic analysis and discontinuous groups in weakly symmetric Riemannian spaces with applications to Dirichlet series, J. Indian Math. Soc. 20 (1956), 47-87. MR 19:531g

[10] D. Sullivan, The density at infinity of a discrete group of hyperbolic motions, Publ. Math. IHES 50 (1979), 171-202. MR 81b:58031

[11] P. Tukia, The Hausdorff dimension of the limit set of a geometrically finite Kleinian group, Acta Math. 152 (1984), 125-140. MR 85m:30031

Department of Mathematics, Aristotle University of Thessaloniki, 54006 ThessaLONIKI, GREECE 